\title{
Composite Hemangioendothelioma of the Submandibular Region
}

\author{
Sarah Lam Shang Leen • Peter M. Clarke • \\ John Chapman · Cyril Fisher · Khin Thway
}

Received: 7 January 2015/Accepted: 29 January 2015/Published online: 10 February 2015

(C) Springer Science+Business Media New York 2015

\begin{abstract}
Composite hemangioendothelioma (HE) is a rare vascular neoplasm of intermediate malignant potential that predominantly occurs within the dermis or subcutis of the extremities, and occurs in a wide age range. It is locally aggressive with a high rate of local recurrence, and more rarely regional lymph node or distant metastasis. Histologically, it is composed of a complex admixture of benign, intermediate and malignant vascular components. Although composite HE may contain angiosarcoma-like areas, its prognosis is better than that of pure angiosarcoma. We describe a case of composite HE presenting as a submandibular mass in a 43 year-old male, which included areas of prominent 'high grade' epithelioid angiosarcoma. This adds to the range of anatomic sites of these neoplasms, highlights the importance of recognition of the head and neck as a potential site, and emphasizes the importance of accurate diagnosis for correct management (including of long term follow up) and prognostication.
\end{abstract}

Keywords Composite hemangioendothelioma $\cdot$ Head and neck · Soft tissue tumor

S. L. S. Leen · C. Fisher $\cdot$ K. Thway $(\bowtie)$

Sarcoma Unit, The Royal Marsden NHS Foundation Trust, 203

Fulham Road, London SW3 6JJ, UK

e-mail: khin.thway@rmh.nhs.uk

P. M. Clarke - C. Fisher - K. Thway

Head and Neck Unit, The Royal Marsden NHS Foundation

Trust, London, UK

J. Chapman

Department of Histopathology, Ipswich Hospital, Ipswich, UK

\section{Introduction}

Composite hemangioendothelioma (HE) is a rare vascular neoplasm of intermediate malignant potential [1] which is characterized by a variable mixture of histologically benign, low grade malignant and malignant vascular components, with merging of the different patterns. Fewer than 40 cases are described in the literature [2-25], with over half occurring in the dermis or subcutis of the extremities. We describe a case arising in the submandibular area with direct extension into a lymph node, which occurred in a 43 year old male. We also review the clinical and pathologic features of composite HE of the head and neck region. This case adds to the number of composite HE reported at non-extremity soft tissue sites, and highlights their relative frequency in the head and neck, and the importance of diagnostic recognition in order to ensure correct management and prognostication.

\section{Case History}

A 43 year old Caucasian male presented with a 3 month history of a firm swelling in the left submandibular area. He had a past medical history of hypertension, but was otherwise well, without history of any pre-existing vascular malformation, clotting disorder, irradiation or immunocompromise. Magnetic resonance imaging of the neck showed a slightly lobulated, rim-enhancing $2.2 \mathrm{~cm}$ diameter soft tissue mass abutting the anterior aspect of the left submandibular gland. There was slightly prominent nodal tissue lying anterior to the inferior right submandibular gland, but no other abnormality. Imaging of the trunk was unremarkable apart from several benign calcific foci in the right lung. Core biopsy of the mass (at the region 
of level I lymph nodes) was performed, which showed a malignant epithelioid vascular tumor with differential diagnosis between angiosarcoma and composite HE. The mass was excised via left level I neck dissection, as well as excisions of left level II and the subcutaneous margin.

On gross examination, the main specimen consisted of a $50 \times 60 \times 35 \mathrm{~mm}$ piece of fibromuscular and fatty tissue, with one side distended by a nodule. Sectioning showed a $20 \times 25 \times 25 \mathrm{~mm}$ circumscribed, firm pale nodule with adjacent salivary gland. Two separate smaller specimens comprised fat measuring $20 \times 5 \times 4 \mathrm{~mm}$, and $50 \times 15 \times 10 \mathrm{~mm}$ with two lymph nodes (measuring 27 and $15 \mathrm{~mm}$ in length). Histologically, the fibromuscular tissue contained a partially circumscribed nodule with an infiltrative edge (Fig. 1a), composed of tumor with a complex mixture of spindle cell hemangioma, retiform and epithelioid HE, and solid angiosarcoma-like areas. The spindle cell hemangioma areas were composed of sheets and long fascicles of bland, elongated spindle endothelial cells with slit-like vascular spaces (Fig. 1b). Within the retiform areas there were elongated thin walled vascular channels lined by endothelial cells forming a hobnail pattern (Fig. 1c). Also scattered throughout the neoplasm were epithelioid cells with large intracytoplasmic vacuoles (Fig. 1d). The blander elements of the neoplasm were admixed with prominent higher grade foci (Fig. 1e-h). Epithelioid hemangioendothelioma (EHE)-like areas comprised cords and nests of polygonal endothelial cells with rounded nuclei showing moderate pleomorphism, and abundant eosinophilic cytoplasm. These were present within hyalinized or focally myxoid stroma (Fig. 1g), with areas of red cell extravasation. The angiosarcoma-like areas were most frequently present at the periphery of the neoplasm, and showed variable morphology (Fig. 1e, f, h). Some comprised lobulated or infiltrative proliferations of well defined vascular channels, lined by mildly to moderately atypical spindle endothelial cells, some with prominent nucleoli (Fig. 1e-f), while others areas were composed of nests of large, highly pleomorphic epithelioid cells, with vesicular nuclei, prominent large nucleoli (Fig. 1h) and a mitotic index of up to $25 / 10$ high power fields (in contrast to the paucity of mitotic figures in the remainder of the neoplasm). No necrosis or lymphovascular invasion was identified. A peripheral area of nodal tissue surrounded the tumor, in keeping with direct invasion of the neoplasm into an adjacent lymph node. Nine further lymph nodes contained no tumor. The tumor did not invade the submandibular gland, which was unremarkable. Immunohistochemically, the neoplasm was diffusely positive for CD31, ERG and D2-40, and focally positive for CD34. MIB1 labelled up to $90 \%$ of lesional nuclei at the periphery of the tumor in the angiosarcoma-like areas, although only approximately $5-10 \%$ in the rest of the neoplasm. The tumor was negative for HHV8 and AE1/ AE3 (which was negative in all components, including EHE-like and epithelioid angiosarcoma-like areas). The features were of composite HE containing 'high grade' epithelioid angiosarcoma-like areas. As the neoplasm had been completely excised macroscopically, no adjuvant treatment was given, although the patient remains under close clinical observation and is well 8 months after surgery.

\section{Discussion}

We describe a case of composite HE arising in the submandibular area of a 43 year old male. Composite HE occurs in a wide age range, is slightly more common in females (24 females, 15 males including this case) and most patients report a long history of having had the lesion (months to years) prior to presentation. The etiology of composite HE is unknown, but it has been described in association with vascular abnormalities, such as lymphangioma circumscriptum [2], Mafucci syndrome (multiple enchondromas and vascular tumors) [9], previous radiation therapy and longstanding lymphedema [20], and consumptive coagulopathy (Kasabach-Merritt syndrome) [9].

While over half of the reported cases (22/38) have occurred within the dermis or subcutis of the extremities, the next most frequent site has been the head and neck region, with cases also reported in the mediastinum [14], manubrium sterni [22], and viscera such as the kidney [23] and spleen [17]. Of composite HE of the head and neck, tumors have been described on the face [18], neck [19], scalp [21, 24], oral cavity $[2,11]$, hypopharynx and mandibular vestibule [9] (Table 1). While this is the tenth reported case of composite HE arising within the head and neck, it is the first to be described at this submandibular site.

Composite HE are poorly defined lesions that are usually solitary, and less frequently multiple. They can occur as dermal, or sometimes more deeply extending papules, nodules or ill-defined swellings, and range in appearance from flesh colored, red, purple or black violaceous lesions that can be scaly or hemorrhagic. There is a wide clinical differential diagnosis, which ranges from viral wart to an extensively erythematous maculopapular lesion suggestive of angiosarcoma. No tumor-related deaths have been reported in association with composite HEs, although approximately $50 \%$ recur locally and about $15 \%$ of lesions metastasize, predominantly to regional lymph nodes $[2,7$, $10,15,24]$, while distant metastasis is exceptional [2, 7]. Local recurrence can occur up to 10 years after initial excision, highlighting the necessity of long term follow up [2]. Due to the rarity of composite HE, its optimal treatment is not well established. The majority of reported 

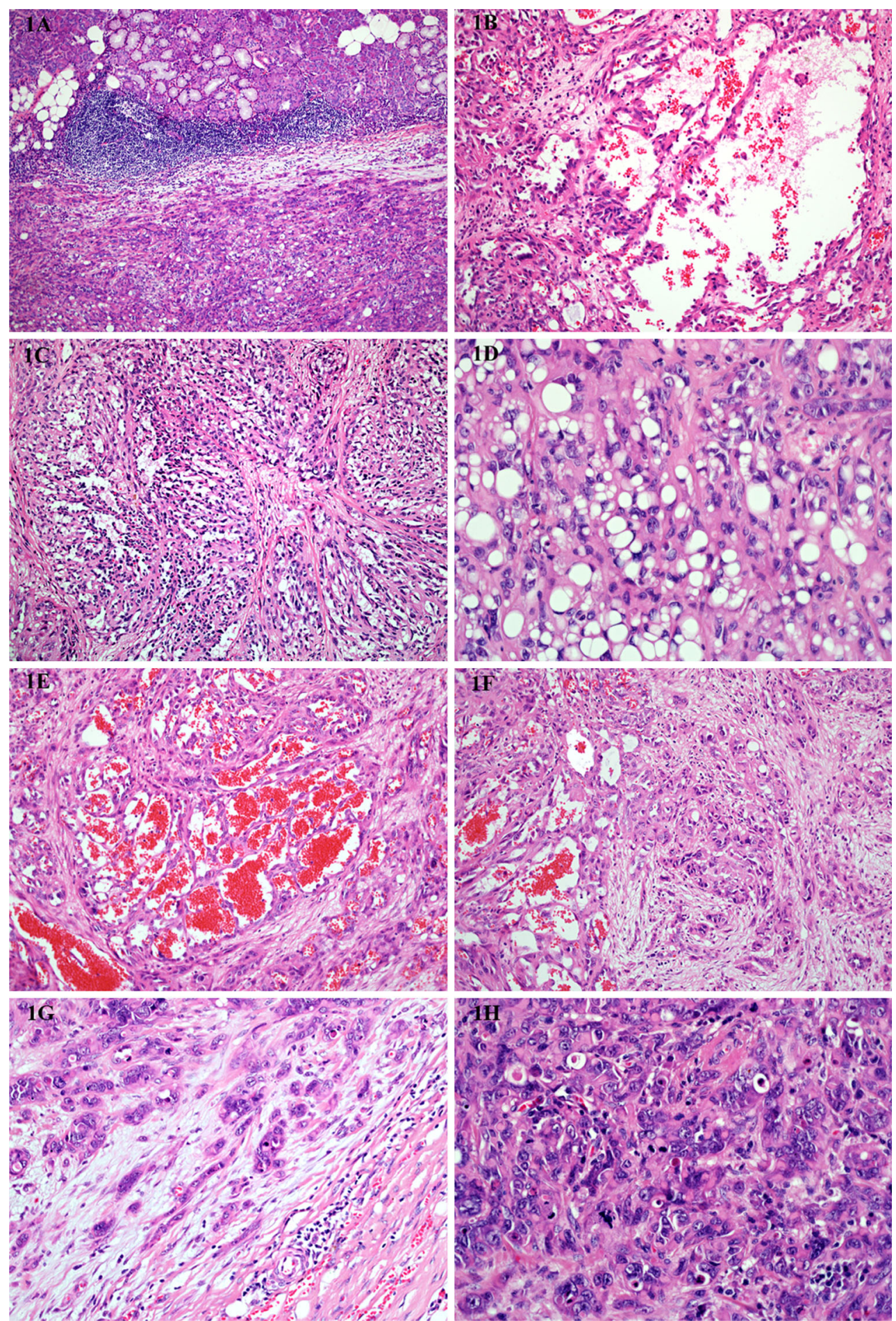
4Fig. 1 Composite hemangioendothelioma of the submandibular region. a The tumor is seen adjacent to salivary gland parenchyma. Although in many places it is relatively well defined, here it has an irregular, infiltrative border. b Well formed vascular channels are apparent in many areas. This field shows areas with features of cavernous and spindle cell hemangioma, with dilated or slit-like vascular spaces that are lined by bland, elongated spindle endothelial cells. c Also prominent are many areas of retiform hemangioendothelioma, in which elongated thin walled vascular channels are lined by endothelial cells lacking atypia, and often forming a hobnail pattern. d Epithelioid cells with large intracytoplasmic vacuoles are also scattered throughout the neoplasm. e This area shows well differentiated angiosarcoma. While vascular lumina are well formed in this component and it has a lobulated architecture, the cellular atypia, with enlarged, hyperchromatic lining endothelial cells is apparent even at low power. More poorly differentiated angiosarcomatous areas are also present (bottom right of field). f The complex mixture of elements is notable, with better-differentiated vascular elements (left) adjacent to those of more high grade appearance (right). g EHE-like areas are also present, and comprise cords and nests of polygonal endothelial cells with rounded, moderately pleomorphic nuclei and abundant eosinophilic cytoplasm, within hyalinized or focally myxoid stroma. h High grade epithelioid angiosarcoma-like areas are also interspersed, and are composed of nests of large, highly pleomorphic epithelioid cells, with vesicular nuclei, prominent large nucleoli and frequent mitotic activity

tumors have been treated with surgical excision, with wider excision advised in some cases due to the potential for local recurrence [25].

Composite HE is characterized by complex admixtures of benign, intermediate and malignant vascular components. Benign components commonly include spindle cell hemangioma, although cavernous hemangioma [14, 15], capillary hemangioma [14, 15], lymphangioma [2, 9] and angiomatosis (9) are also described. Usually there are also areas of retiform HE and epithelioid HE, and less commonly foci of Dabska-type HE [10, 11, 19, 21, 25] (papillary intralymphatic angioendothelioma) and kaposiform HE [7, 14, 15]. Angiosarcoma-like areas have been described in approximately half of composite HEs, and the majority are well differentiated, although high grade or epithelioid angiosarcoma-like areas are also reported [2, 5, 9].

Due to the morphologic heterogeneity of composite HE, the differential diagnosis encompasses the individual benign, intermediate and malignant vascular components present in each case. Diagnosis on core biopsy can be challenging due to limited or non-representative sampling, and careful assessment is required to identify the mixture of vascular components and characteristic merging of individual vascular patterns that distinguish composite HE from other vascular tumors. In excision specimens of vascular neoplasms, it is important to adequately sample different areas to ensure that the diagnosis of composite HE is not overlooked. It is also essential to differentiate composite HE from angiosarcoma, as although composite HE may contain angiosarcoma-like areas, they have a better prognosis than pure angiosarcomas of soft tissue [26].

Composite HE needs to be distinguished from a number of vascular lesions that are more specifically associated with the head and neck region. It is important to differentiate composite HE from angiosarcoma because of the marked prognostic difference between the two entities. While angiosarcomas also arise predominantly cutaneously within the head and neck region, they usually occur within elderly patients, particularly on the scalp and face, where they are associated with sun exposure. Epithelioid angiosarcoma shows diffuse rather than localized nuclear atypia and more marked mitotic activity. Epithelioid HE also has a propensity for the head and neck region, but may present multicentrically and more frequently involves deep soft tissues, solid organs and bone [27, 28], while primary presentation in the skin is rare [29]. About half of EHE are associated with a medium-sized or large vessel. In addition to expressing vascular markers, EHE can express cytokeratins, and are associated with WWTRI-CAMTAl [30] or YAP1-TFE3 [31] fusions, which have not yet been shown to be associated with other neoplasms. Kaposi sarcoma (KS) may be associated with immunosuppression, or with certain populations (e.g. classical indolent form in elderly Eastern European or Mediterranean males, or endemic form in children and middle aged adults in equatorial Africa). Histologically, KS can show slit- and sieve-like spaces containing erythrocytes or hyaline globules representing degenerate erythrocytes, and expresses nuclear HHV8 immunohistochemically. Epithelioid hemangioma (angiolymphoid hyperplasia with eosinophilia) commonly arises in the head and neck, particularly around the ear, and comprises a lobular capillary proliferation that often surrounds a central vessel, with prominent dense lymphoid aggregates and numerous eosinophils. It lacks a complex vascular architecture and nuclear pleomorphism.

In summary, this is the first case of composite $\mathrm{HE}$ occurring at a submandibular site, which adds to the spectrum of composite HE described at non-extremity sites as well as the increasing number of cases reported in the head and neck region. This highlights the importance of diagnostic recognition of composite HE within the head and neck, both for prognostic reasons, as it tends to behave much more indolently compared to morphologic mimics such as angiosarcoma and EHE, but also to ensure long term follow up, as composite HE can recur and metastasize after long periods. 


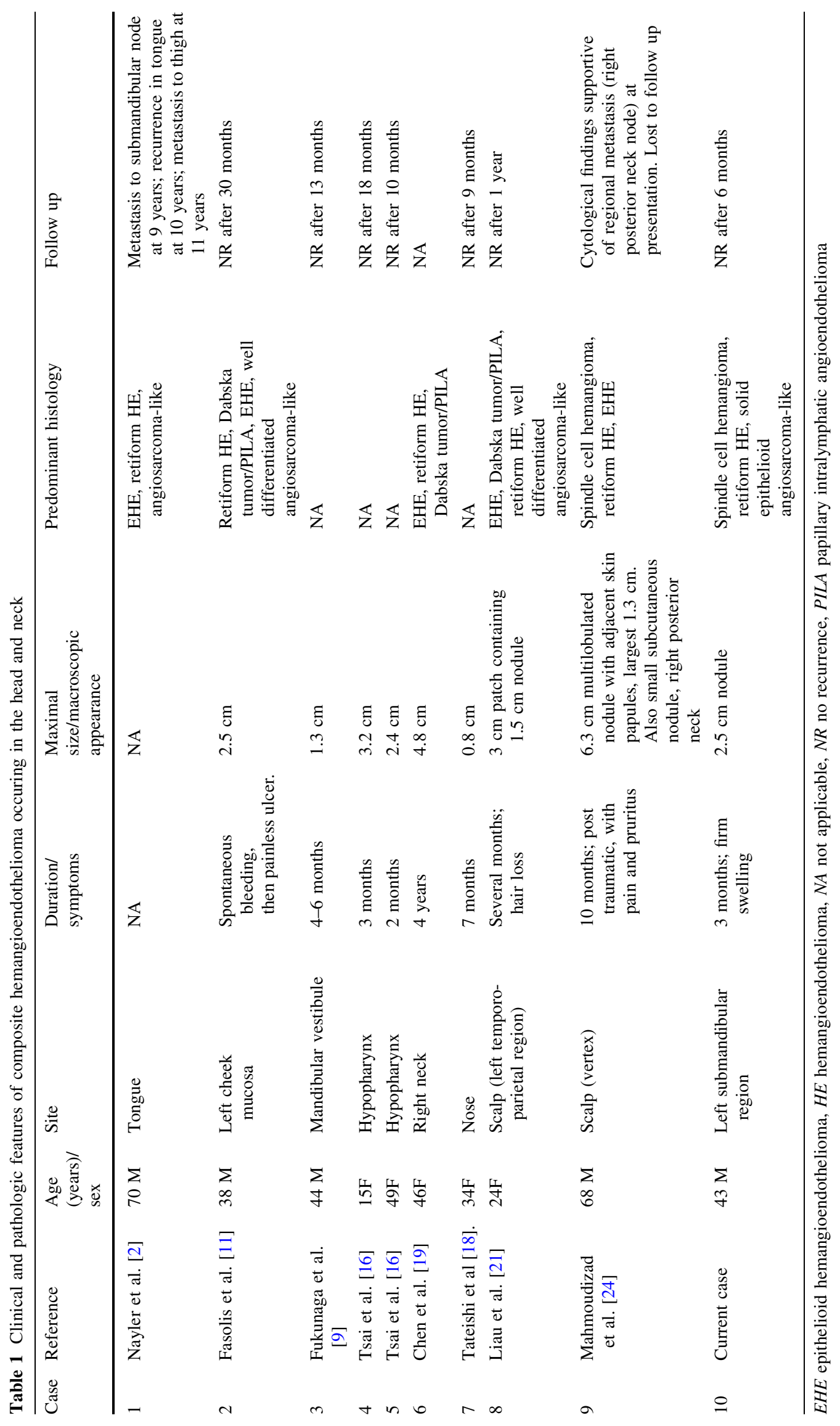


Acknowledgments We acknowledge support from the NIHR Royal Marsden/ICR Biomedical Research Centre.

Conflict of interest The authors have no conflicts of interest or funding to disclose.

\section{References}

1. Rubin BP. Composite haemangioendothelioma. In: Fletcher CDM, Bridge JA, Hogendoorn PCW, Mertens F, editors. WHO Classification of Tumours of Soft Tissue and Bone. 4th ed. Lyon: IARC; 2013. p. 149-50.

2. Nayler SJ, Rubin BP, Calonje E, Chan JK, Fletcher CD. Composite hemangioendothelioma: a complex, low-grade vascular lesion mimicking angiosarcoma. Am J Surg Pathol. 2000;24:352-61.

3. Silva EG, Phillips MJ, Langer B, Ordonez NG. Spindle and histiocytoid (epithelioid) hemangioendothelioma. Primary in lymph node. Am J Clin Pathol. 1986;85:731-5.

4. Zoltie N, Roberts PF. Spindle cell haemangioendothelioma in association with epithelioid haemangioendothelioma. Histopathology. 1989;15:544-6.

5. Reis-Filho JS, Paiva ME, Lopes JM. Congenital composite hemangioendothelioma: case report and reappraisal of the hemangioendothelioma spectrum. J Cutan Pathol. 2002;29:226-31.

6. Sapunar J, Roa JC, Moscoso S. Reversion of hypophosphatemia after the excision of a composite hemangioendothelioma in the great toe. Rev Med Chile. 2003;131:909-14.

7. Chu YC, Choi SJ, Park IS, Kim L, Han JY, Kim JM. Composite haemangioendothelioma- a case report. Korean J Pathol. 2006;40:142-7.

8. Tronnier M, Vogelbruch M, Kutzner H. Spindle cell hemangioma and epithelioid hemangioendothelioma arising in an area of lymphedema. Am J Dermatopathol. 2006;28:223-7.

9. Fukunaga M, Suzuki K, Saegusa N, Folpe AL. Composite hemangioendothelioma: report of 5 cases including one with associated Maffucci syndrome. Am J Surg Pathol. 2007;31:1567-72.

10. Requena L, Luis Diaz J, Manzarbeitia F, Carrillo R, FernandezHerrera I, Kutzner H. Cutaneous composite hemangioendothelioma with satellitosis and lymph node metastases. J Cutan Pathol. 2008;35:225-30.

11. Fasolis M, Iaquinta C, Montesco MC, et al. Composite hemangioendothelioma of the oral cavity: case report and review of the literature. Head Neck. 2008;30:974-9.

12. Utas S, Canoz O, Ferahbas A, Ozcan N. Composite cutaneous haemangioendothelioma treated with interferon. J Eur Acad Dermatol Venereol. 2008;22:503-5.

13. Tejera-Vaquerizo A, Herrera-Ceballos E, Bosch-Garcia R, Fernandez-Orland A, Matilla A. Composite cutaneous hemangioendothelioma on the back. Am J Dermatopathol. 2008;30:262-4.

14. Cakir E, Demirag F, Gulhan E, Oz G, Tastepez I. Mediastinal composite hemangioendothelioma. A rare tumor at an unusual location. Tumori. 2009;95:98-100.
15. Aydingoz IE, Demirkesen C, Serdar ZA, Mansur AT, Yasar S, Aslan C. Composite haemangioendothelioma with lymph-node metastasis: an unusual presentation at an uncommon site. Clin Exp Dermatol. 2009;34:e802-6.

16. Tsai JW, Huang HY, Lee JC, et al. Composite haemangioendothelioma: report of four cases with emphasis on atypical clinical presentation. Pathology. 2011;43:176-80.

17. Yoda Y, Ohashi M. A case of composite hemangioendothelioma arising from the spleen. Jpn J Clin Oncol. 2012;42:770.

18. Tateishi J, Saeki H, Ito K, Nakagawa H, Fukunaga M. Cutaneous composite hemangioendothelioma on the nose treated with electron beam. Int J Dermatol. 2013;52:1618-9.

19. Chen YL, Chen WX, Wang J, Jiang Y. Composite hemangioendothelioma on the neck. Kaohsiung $\mathbf{J}$ Med Sci. 2012;28:564-5.

20. McNab PM, Quigley BC, Glass LF, Jukic DM. Composite hemangioendothelioma and its classification as a low-grade malignancy. Am J Dermatopathol. 2013;35:517-22.

21. Liau JY, Lee FY, Chiu CS, Chen JS, Hsiao TL. Composite hemangioendothelioma presenting as a scalp nodule with alopecia. J Am Acad Dermatol. 2013;69:e98-9.

22. Dong A, Bai Y, Wang Y, Zuo C. Bone scan, MRI, and FDG PET/ CT findings in composite hemangioendothelioma of the manubrium sterni. Clin Nucl Med. 2014;39:e180-3.

23. Zhang J, Wu B, Zhou GQ, et al. Composite hemangioendothelioma arising from the kidney: case report with review of the literature. Int J Clin Exp Pathol. 2013;6:1935-41.

24. Mahmoudizad R, Samrao A, Bentow JJ, Peng SK, Bhatia N Composite hemangioendothelioma: an unusual presentation of a rare vascular tumor. Am J Clin Pathol. 2014;141:732-6.

25. Biagioli M, Sbano P, Miracco C, Fimiani M. Composite cutaneous haemangioendothelioma: case report and review of the literature. Clin Exp Dermatol. 2005;30:385-7.

26. Meis-Kindblom JM, Kindblom LG. Angiosarcoma of soft tissue: a study of 80 cases. Am J Surg Pathol. 1998;22:683-97.

27. Mentzel T, Beham A, Calonje E, Katenkamp D, Fletcher CD. Epithelioid hemangioendothelioma of skin and soft tissues: clinicopathologic and immunohistochemical study of 30 cases. Am J Surg Pathol. 1997;21:363-74.

28. Weiss SW, Ishak KG, Dail DH, Sweet DE, Enzinger FM. Epithelioid hemangioendothelioma and related lesions. Semin Diagn Pathol. 1986;3:259-87.

29. Goh SG, Calonje E. Cutaneous vascular tumours: an update. Histopathology. 2008;52:661-73.

30. Errani C, Zhang L, Sung YS, et al. A novel WWTR1-CAMTA1 gene fusion is a consistent abnormality in epithelioid hemangioendothelioma of different anatomic sites. Genes Chromosomes Cancer. 2011;50(8):644-53.

31. Antonescu CR, Le Loarer F, Mosquera JM, et al. Novel YAP1TFE3 fusion defines a distinct subset of epithelioid hemangioendothelioma. Genes Chromosomes Cancer. 2013;52:775-84. 